Molecules 2008, 13, 1472-1486; DOI: 10.3390/molecules13071472

www.mdpi.org/molecules

Article

\title{
Facile Synthesis of Oleanolic Acid Monoglycosides and Diglycosides
}

\author{
Yu Sha, Mao-Cai Yan, Jiao Liu, Yang Liu and Mao-Sheng Cheng *
}

Key Lab of New Drug Design and Discovery of Liaoning Province, School of Pharmaceutical Engineering, Shenyang Pharmaceutical University, Shenyang 110016, P. R. China; E-mails: syna2000@yahoo.com (Y.S.); yanmaocai@126.com (M.-C. Y.); amityliu@163.com (Y. L.); 1y_99@sina.com (Y. L.)

* Author to whom correspondence should be addressed: E-Mail: mscheng@263.net

Received: 16 June 2008; in revised form: 18 July 2008 / Accepted: 18 July 2008 / Published: 22 July 2008

Abstract: Oleanolic acid and its glycosides are important natural products, possessing various attractive biological activities such as antitumor, antivirus and anti-inflammatory properties. In the present work, fifteen oleanolic acid saponins bearing various saccharide moieties, including 3-monoglycoside, 28-monoglycoside and 3,28-diglycoside, were easily synthesized in high yields. Benzyl was chosen as the protective group for the $\mathrm{COOH}(28)$ group, instead of commonly used methyl and allyl, to avoid difficulties in the final deprotection. Alkali-promoted condensation of the carboxylic acid with bromoglycosides was found to be more efficient in the synthesis of 28 -glycosides. Two approaches were investigated and proved practicable in the preparation of 3,28diglycosides. This method is suitable for preparing oleanolic acid glycosides with structural diversity for extensive biological evaluation and structure-activity relationship study, and it also apply new idea for the corresponding synthetic methods to the glycoside derivatives of other triterpenoid.

Keywords: Oleanolic acid, triterpenoid, glycoside, synthesis, glycosylation 


\section{Introduction}

Oleanolic acid (OA, 1, Figure 1) is one of the most important triterpenes, which is found widely distributed in Nature, along with its glycosides [1]. Possessing multiple biological effects, such as antitumor, antiulcer, antivirus, antihyperlipidemic, anti-inflammatory and hepatoprotective activities [1-5], OA and its saponins have received increasing attentions in recent years. The saccharide moiety of OA saponins has been revealed to be of importance for their bioactivities. For example, oleananetype triterpenoid saponins bearing an $\alpha$-L-rhamnopyranosyl- $(1 \rightarrow 2)-\alpha$-L-arabinopyranosyl moiety at $\mathrm{C}(3)-\mathrm{OH}$ generally show markedly higher antitumor activity than their aglycons $[4,5]$. Some OA glycosides show significant gastrointestinal transit acceleration or inhibition activity in mice, while the aglycon OA does not [6].

Although OA has become an auxiliary drug in the treatment of liver disease, many bioactivities of OA glycosides are too weak to be utilized in clinical therapy. Chemical synthesis and modification is known to be a powerful tool in preparation of novel compounds with diversity for pharmacology studies and new chemical entity development. In fact, the synthesis of OA glycosides has attracted much attention from researchers for a long time. As early as 1952, Hardegger et al. reported the glycosylation of OA esters with acetylated bromoglycosides [7]. Later a few studies were reported on the glycosylation of OA by the Koenigs-Knorr method [8-13], which was usually inefficient and resulted in uneven yields. Although methyl was most commonly used as protective group for the carboxylic acid in the early synthesis work, alkaline hydrolysis of OA esters was very difficult due to the high steric hinderance [14]. Therefore, halolysis (usually LiI/DMF, reflux) was often employed to cleave OA esters, but was of low efficiency and too harsh for many groups. In 1999, Deng et al. reported a highly efficient glycosylation of triterpenoids and steroids via the Schmidt method [15, 16], in which the allyl ester of OA was glycosylated with benzoylated trichloroacetimidates in very high yields (>90\%). Based on this method, Zang et al. accomplished in 2005 the synthesis of four 3-monoglycosides of OA [17]. The glycosylation of allyl oleanolate was carried out in $80 \sim 90 \%$ yield, however, removal of allyl in the final step by treatment with $\mathrm{PdCl}_{2}$ for $24 \mathrm{~h}$ afforded relatively lower yields (only 41 47\%). A few syntheses of some bioactive OA saponins bearing complex saccharide moieties were accomplished in recent years [18-20], nevertheless more facile and efficient synthetic methods towards this interesting structure require further investigation. Based on our experience in triterpenoid saponin synthesis, we started with the highly efficient preparation of OA glycosides on a large scale for extensive bioactivity evaluation and structure-activity relationship study.

Figure 1. Structure of oleanolic acid

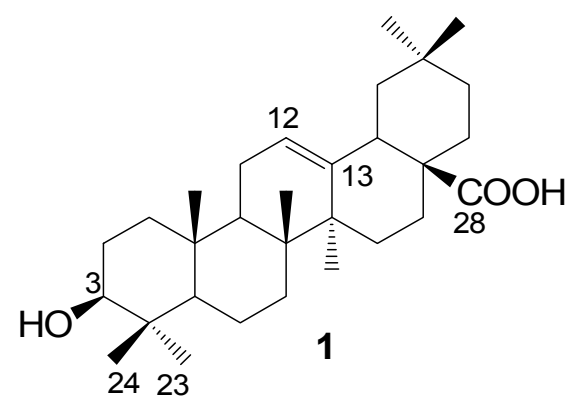




\section{Results and Discussion}

OA has two glycosylation sites, i.e. $\mathrm{C}(3)-\mathrm{OH}$ and $\mathrm{COOH}(28)$, which can be glycosylated to form 3-monoglycosides, 28-monoglycosides and 3,28-diglycosides. The present work describes the initial glycosylation study of OA, which was carried out with some commercially available sugars, such as Dglucose, L-arabinose, lactose and maltose.

\section{Preparation of $O A$ 3-glycosides}

Since common alkyl esters of OA are difficult to retransform into free carboxylic acids, selection of a suitable protective group for $\mathrm{COOH}(28)$ is the primary problem. Trityl (Tr) and tert-butyldiphenylsilyl (TBDPS) had been used in the previous work [21, 22], however, they are too sensitive to act as permanent protective groups in the preparation of complex glycosides. As mentioned above, the deprotection was usually inefficient when allyl was employed [17, 18]. In our previous work on triterpenoid saponin synthesis [19, 23], benzyl was chosen and proved to be an excellent permanent protective group for $\mathrm{COOH}(28)$, as it can be conveniently removed through catalytic hydrogenolysis in nearly quantitive yields, while the double bond between C(12) and C(13) would not be affected [24]. Therefore, we adopted benzyl in the present work.

Scheme 1. Synthesis of OA 3-glycosides.
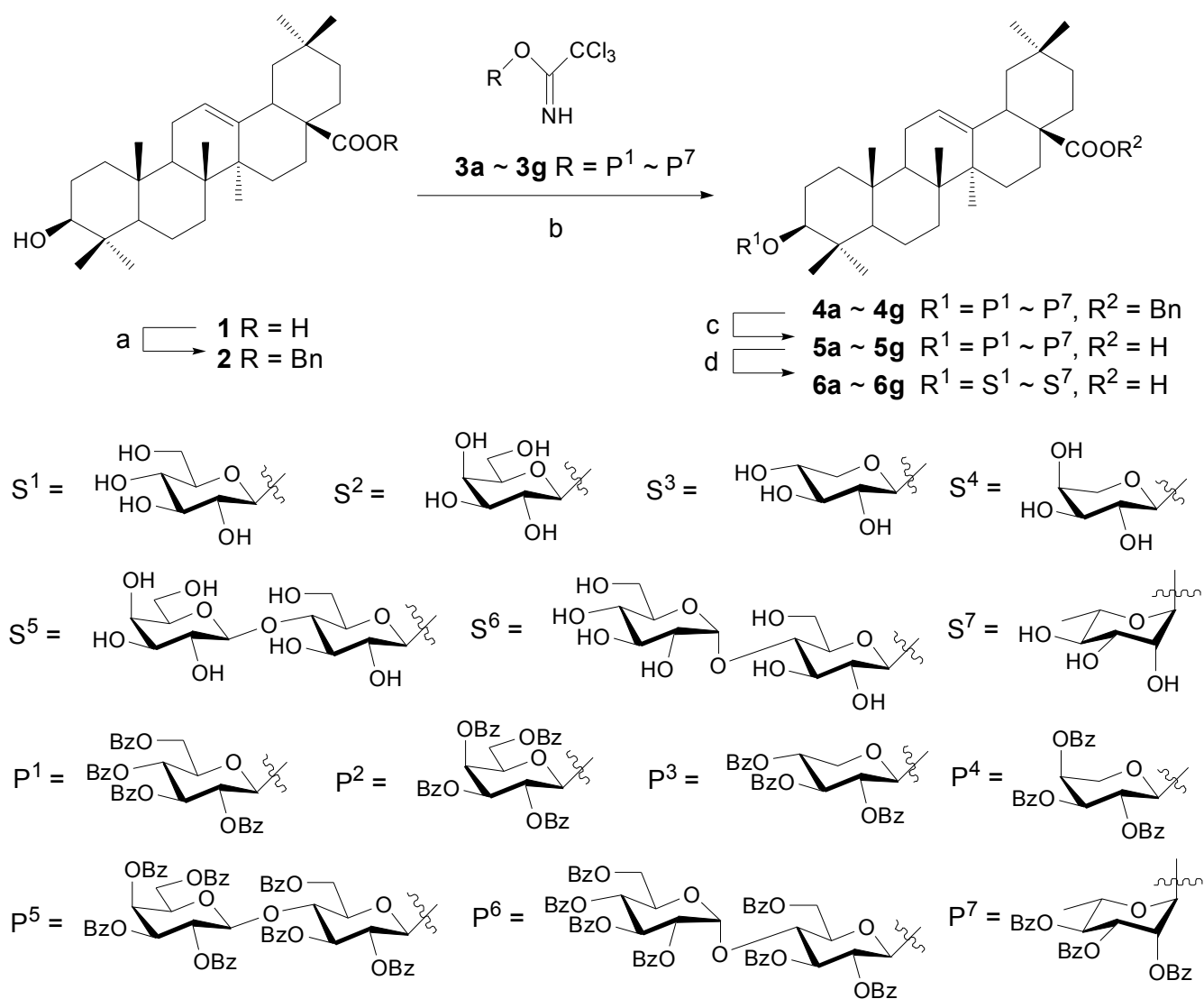

Reagents and conditions: (a) $\mathrm{BnBr}, \mathrm{K}_{2} \mathrm{CO}_{3}$, THF- $\mathrm{H}_{2} \mathrm{O}$, rt; (b) TMSOTf, $4 \AA \mathrm{MS}, \mathrm{CH}_{2} \mathrm{Cl}_{2}$, rt; (c) $\mathrm{H}_{2}$, Pd-C (10\%), EtOAc, reflux; (d) NaOMe, $\mathrm{CH}_{2} \mathrm{Cl}_{2}-\mathrm{MeOH}$, rt. 
As shown in Scheme 1, OA was first converted into its benzyl ester 2 in 98\% yield [23], and C(3)$\mathrm{OH}$ was then glycosylated with trichloroacetimidates $\mathbf{3}$ under promotion with TMSOTf. The benzyl moiety was then removed under catalytic hydrogenolysis to give free carboxylic acids $\mathbf{5}$, which would be subjected to further glycosylation to give 3,28-diglycosides in the later work. Removal of benzoyl on 5 through ester exchange in $\mathrm{NaOMe}-\mathrm{MeOH}$ afforded target OA 3-glycosides $\mathbf{6}$. According to this method, seven 3-glycosides of OA were easily prepared in overall yields of 58\% 79\%.

\section{Preparation of $O A$ 28-glycosides}

Two approaches were attempted to prepare OA 28-glycosides. In the first approach, the C(3)-OH of $\mathrm{OA}$ was acetylated in $\mathrm{Ac}_{2} \mathrm{O}$-pyridine and glycosylation of $\mathrm{COOH}(28)$ was carried out with benzoylated trichloroacetimidates. Benzoyl and acetyl groups were then removed by $\mathrm{NaOMe}-\mathrm{MeOH}$ to give OA 28-glycosides (Scheme 2). However, removal of Ac on $\mathrm{C}(3)-\mathrm{OH}$ was found to be more difficult than that of benzoyl groups on the saccharide moieties due to the steric hinderance from $\mathrm{C}(23)$ and $C(24)$. Stronger alkaline condition usually led to the partial breakage of acyl glycoside linkage at $\mathrm{C}(28)$. By this approach OA ester of $\beta$-D-galactose (9a) and $\alpha$-L-rhamnose (9b) were synthesized in $18 \%$ and $21 \%$ total yields, respectively.

Scheme 2. Synthesis of OA 28-glycosides (the first approach).

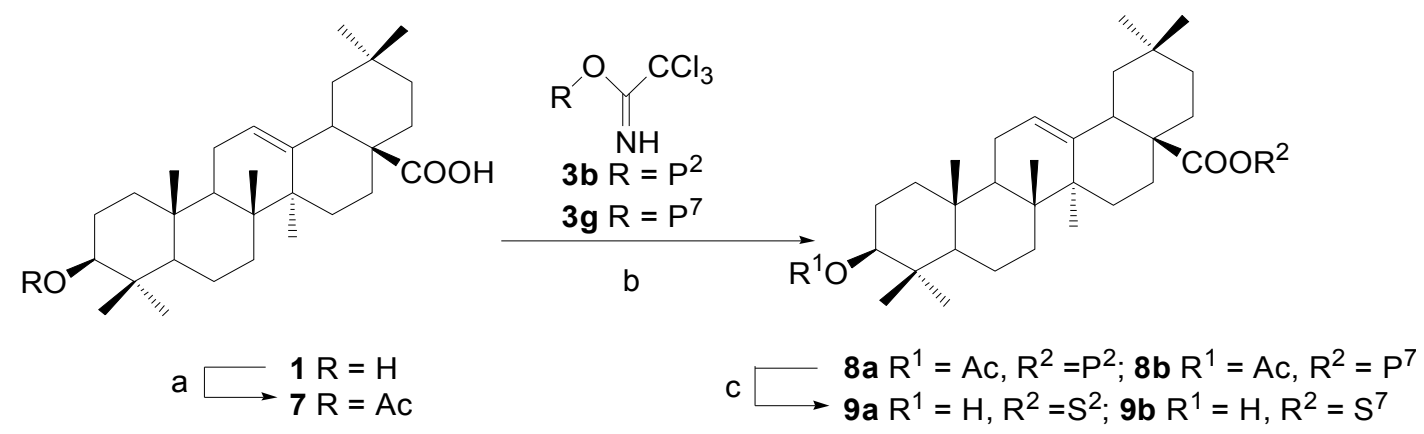

Reagents and conditions: (a) $\mathrm{Ac}_{2} \mathrm{O}$, pyridine, rt; (b) TMSOTf, $4 \AA \mathrm{MS}, \mathrm{CH}_{2} \mathrm{Cl}_{2}, 0^{\circ} \mathrm{C}$; (c) $\mathrm{NaOMe}$, $\mathrm{CH}_{2} \mathrm{Cl}_{2}-\mathrm{MeOH}$, rt.

Scheme 3. Synthesis of OA 28-glycosides (the second approach).
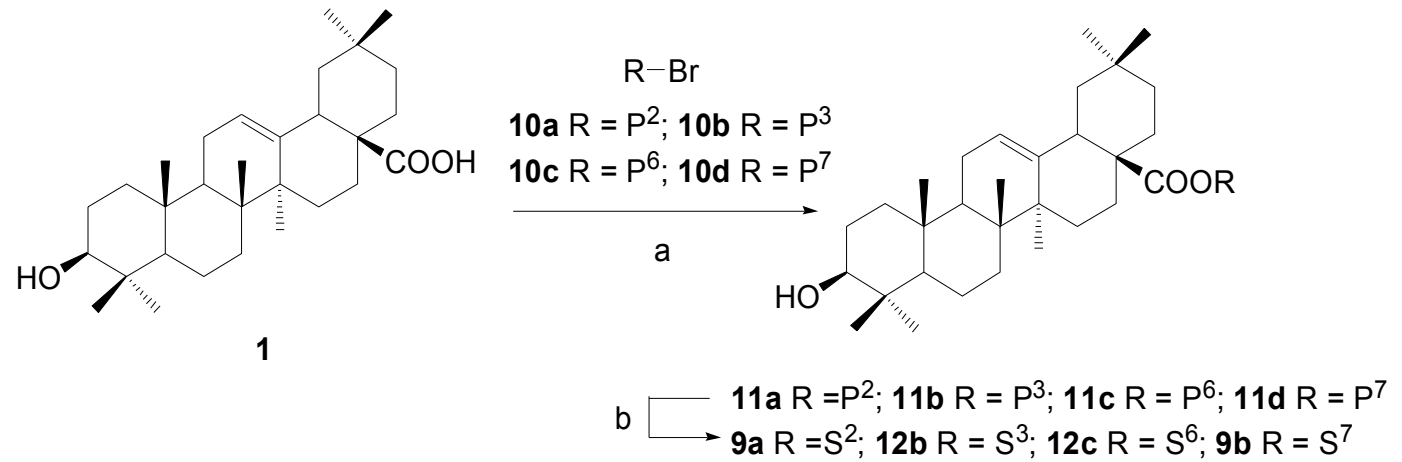

Reagents and conditions: (a) $\mathrm{K}_{2} \mathrm{CO}_{3}, \mathrm{CHCl}_{3}-\mathrm{H}_{2} \mathrm{O}, \mathrm{Bu}_{4} \mathrm{NBr}, 50^{\circ} \mathrm{C}$; (b) $\mathrm{NaOMe}, \mathrm{CH}_{2} \mathrm{Cl}_{2}-\mathrm{MeOH}$, rt. 
Alternatively, alkaline-promoted condensation of free OA with benzoylated bromoglycosides (R$\mathrm{Br}, \mathrm{R}=\mathrm{P}^{2}, \mathrm{P}^{3}, \mathrm{P}^{6}$, and $\mathrm{P}^{7}$ ) under phase transfer catalysis was attempted (Scheme 3) [25]. However, no significant reaction was observed under the reported conditions, possibly because benzoylated bromoglycosides are more stable than the acetylated donors employed in the literature. When modified conditions $\left(\mathrm{K}_{2} \mathrm{CO}_{3}, \mathrm{Bu}_{4} \mathrm{NBr}, \mathrm{CH}_{2} \mathrm{Cl}_{2}-\mathrm{H}_{2} \mathrm{O}\right.$, reflux) were used [22], the reaction proceeded very slowly and the decomposed bromoglycoside was the main by-product mixed with the desired compound. As improvements, the solvent dichloromethane was replaced by chloroform and the turbid system was heated up to $50^{\circ} \mathrm{C}$ and stirred vigorously to successfully give the glycosylation products. All the benzoyl groups on the sugar parts were removed by $\mathrm{NaOMe} / \mathrm{MeOH}$, while the acyl glycoside linkages were not affected under these conditions. By this approach, glycosides 9a and 9b were synthesized once more in higher yields of $80 \%$ and $78 \%$. Furthermore, other two 28 -glycosides $(\mathbf{1 2 b}, \mathbf{1 2 c})$ were readily prepared from $\mathrm{OA}$ in $74 \%$ and $70 \%$ yields, respectively. Understandably, due to the relatively less efficiency of Schmidt glycosylation of carboxylic acid and low selectivity in the deprotection in the first approach, the second approach was more preferable for the preparation of OA 28-glycosides.

\section{Preparation of $O A$ 3,28-diglycosides}

Many natural occurring saponins of OA with important biological activities are 3,28-diglycosides. Two approaches were also investigated in the preparation of this type of structures. The first approach was to introduce one saccharide moiety at $\mathrm{C}(3)-\mathrm{OH}$ after protection of $\mathrm{COOH}(28)$ with benzyl, and to attach another saccharide moiety to the carboxylic acid after removal of the benzyl. The second approach was to introduce one saccharide to $\mathrm{COOH}(28)$ using a bromoglycoside under alkaline conditions, then $\mathrm{C}(3)-\mathrm{OH}$ was to be glycosylated with a trichloroacetimidate. In the present work, intermediates 5 and $\mathbf{1 1}$ in the preparation of 3-glycosides and 28-glycosides were utilized in the synthesis of OA 3,28-diglycosides through these two approaches, respectively.

Scheme 4. Synthesis of OA 3,28-diglycosides (the first approach).

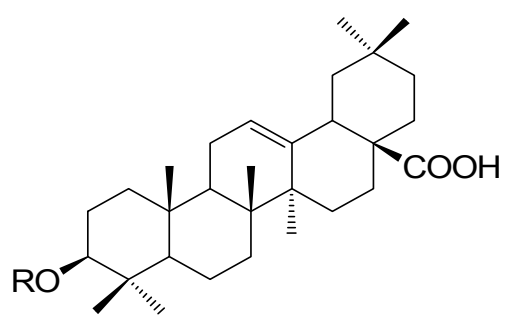

$\mathbf{5 b} \mathrm{R}=\mathrm{P}^{2} ; \mathbf{5 d} \mathrm{R}=\mathrm{P}^{4}$

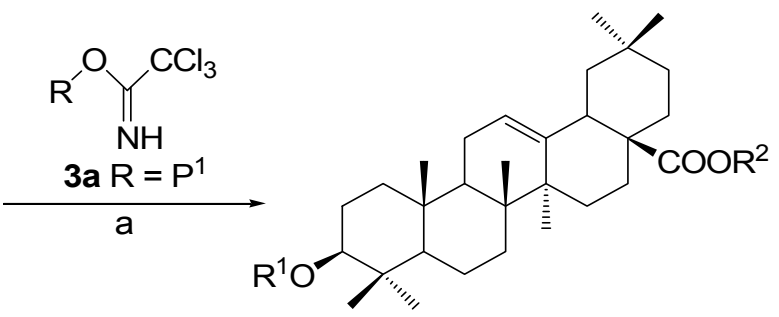

b $\quad$ 13a $R^{1}=P^{2}, R^{2}=P^{1} ; 13 b R^{1}=P^{4}, R^{2}=P^{1}$

14a $R^{1}=S^{2}, R^{2}=S^{1} ; 14 b R^{1}=S^{4}, R^{2}=S^{1}$

Reagents and conditions: (a) TMSOTf, $4 \AA \mathrm{MS}, \mathrm{CH}_{2} \mathrm{Cl}_{2}, 0^{\circ} \mathrm{C} \sim \mathrm{rt}$; (b) $\mathrm{NaOMe}, \mathrm{CH}_{2} \mathrm{Cl}_{2}-\mathrm{MeOH}$, rt.

As shown in Scheme 4, 3- $O$-(2,3,4,6-tetra- $O$-benzoyl- $\beta$-D-galactopyranosyl)oleanolic acid (5b) was subjected to Schmidt glycosylation with trichloroacetimidate 3a to give the full protected 3,28diglycoside 13a, which was then treated with $\mathrm{NaOMe}-\mathrm{MeOH}$ to afford target product $\beta$-D- 
glucopyranosyl oleanolate 3- $O-\beta$-D-galactopyranoside (14a). According to the same procedure, $\beta$-Dglucopyranosyl oleanolate 3-O- $\alpha$-L-arabinopyranoside (14b) was prepared from intermediate 5d.

In the second approach, the intermediate 2,3,4-tri- $O$-benzoyl- $\beta$-D-xylopyranosyl oleanolate (11b) was subjected to Schmidt glycosylation with $\mathbf{3 b}$ to introduce a galactose residue to the free $\mathrm{C}(3)-\mathrm{OH}$ (Scheme 5). Removal of benzoyl groups afforded target $\beta$-D-xylopyranosyl oleanolate $3-O-\beta$-Dgalactopyranoside (16a). According to the same procedure, $\alpha$-D-glucopyranosyl- $(1 \rightarrow 4)-\beta$-Dglucopyranosyl oleanolate 3-O- $\beta$-D-xylopyranoside (16b) was synthesized from intermediate 11c and trichloroacetimidate $\mathbf{3 c}$.

Scheme 5. Synthesis of OA 3,28-diglycosides (the second approach).

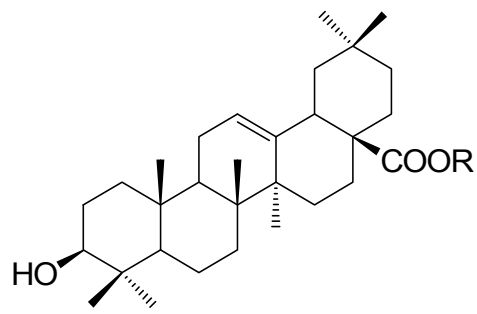

11b $R=P^{3} ; 11 c R=P^{6}$

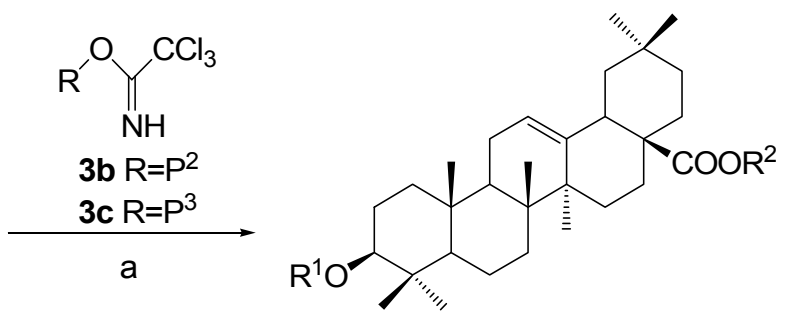

$\mathrm{b}$ 15a $R^{1}=P^{2}, R^{2}=P^{3} ; 15 b R^{1}=P^{3}, R^{2}=P^{6}$

$16 a R^{1}=S^{2}, R^{2}=S^{3} ; 16 b R^{1}=S^{3}, R^{2}=S^{6}$

Reagents and conditions: (a) TMSOTf, $4 \AA \mathrm{AS}, \mathrm{CH}_{2} \mathrm{Cl}_{2}$, rt; (b) $\mathrm{NaOMe}, \mathrm{CH}_{2} \mathrm{Cl}_{2}-\mathrm{MeOH}$, rt.

It is obvious that the second approach to 3,28-diglycosides is preferable to the first one, not only due to its conciseness in avoiding introduction and removal of benzyl group, but also because alkalinepromoted condensation was more efficient in the glycosylation of carboxylic acid. However, the first approach also affords good yields and it is applicable in certain cases. For instance, in the synthesis of glycoside analogues with the same 3-O-saccharide moiety, or in preparation of glycosides in large amounts, it can make use of various intermediates with free carboxylic acids.

The 15 glycosides prepared in the present work and the overall yields from OA are listed in Table 1. All glycosidic linkages formed in the glycosylation are 1,2-trans-, due to the neighboring participating effect of benzoyl at $\mathrm{C}(2)-\mathrm{OH}$ of saccharide donors, which can also be confirmed by the $J$ values of hydrogen on the anomeric carbon of the saccharide moiety. In ${ }^{1} \mathrm{H}-\mathrm{NMR}$ spectra of the synthetic saponins, the 1,2-trans- configuration of the anomeric carbons of all glucosyl, gallactosyl, arabinosyl and xylosyl units were determined by the $J_{\mathrm{H} 1-\mathrm{H} 2}$ values (above $6.0 \mathrm{~Hz}$ ), while the $J_{\mathrm{H} 1-\mathrm{H} 2}$ values $(1.0-1.5$ $\mathrm{Hz}$ ) of all rhamnosyl units indicated the alpha-configuration of their anomeric carbons. For compound 6a, its $7.7 \mathrm{~Hz} J$ value of the hydrogen on anomeric carbon with characterized $4.93 \mathrm{ppm}$ chemical shift indicates that two hydrogens on $\mathrm{C}\left(1^{\prime}\right)$ and $\mathrm{C}\left(2^{\prime}\right)$ of glucose are 1,2-trans-configuration. And in the case of $9 \mathrm{~b}$, its $1.4 \mathrm{~Hz} J$ value of the hydrogen on anomeric carbon (5.43 ppm) also indicates that two hydrogens on $\mathrm{C}\left(1^{\prime}\right)$ and $\mathrm{C}\left(2^{\prime}\right)$ of rhamnose are 1,2-trans-configuration. 
Table 1. OA glycosides and overall yields from OA.

\begin{tabular}{|c|c|c|c|c|}
\hline Compound & $\begin{array}{c}C(3) \\
\text { saccharide }\end{array}$ & $\begin{array}{c}\mathrm{C}(28) \\
\text { saccharide }\end{array}$ & $\begin{array}{l}\text { Total yield } \\
\text { from OA }\end{array}$ & $\begin{array}{c}\text { Literature } \\
\text { yield }\end{array}$ \\
\hline $6 a$ & $\beta$-D-Glc & $\mathrm{H}$ & $58 \%$ & $\begin{array}{l}25 \%[17] \\
36 \%[11]\end{array}$ \\
\hline $6 b$ & $\beta-\mathrm{D}-\mathrm{Gal}$ & $\mathrm{H}$ & $55 \%$ & $24 \%$ [17] \\
\hline $6 c$ & $\beta-\mathrm{D}-\mathrm{Xyl}$ & $\mathrm{H}$ & $71 \%$ & $26 \%[17]$ \\
\hline 6d & $\alpha$-L-Ara & $\mathrm{H}$ & $76 \%$ & $25 \%[17]$ \\
\hline $6 e$ & $\begin{array}{c}\beta \text {-D-Gal- }(1 \rightarrow 4)- \\
\beta-D-G l c\end{array}$ & $\mathrm{H}$ & $75 \%$ & $4.0 \%[13]$ \\
\hline $6 f$ & $\begin{array}{c}\alpha \text {-D-Glc- }(1 \rightarrow 4)- \\
\beta-D-G l c\end{array}$ & $\mathrm{H}$ & $69 \%$ & - \\
\hline $6 g$ & $\alpha-L-R h a$ & $\mathrm{H}$ & $79 \%$ & - \\
\hline $9 \mathbf{a}$ & $\mathrm{H}$ & $\beta$-D-Gal & $\begin{array}{l}18 \% \text { (Scheme } 2) \\
80 \% \text { (Scheme } 3)\end{array}$ & $\begin{array}{c}\text { not presented } \\
{[25]}\end{array}$ \\
\hline $12 \mathrm{~b}$ & $\mathrm{H}$ & $\beta-\mathrm{D}-\mathrm{Xyl}$ & $74 \%$ & $54 \%[10]$ \\
\hline $12 \mathrm{c}$ & $\mathrm{H}$ & $\begin{array}{c}\alpha-D-G l c-(1 \rightarrow 4)- \\
\beta-D-G l c\end{array}$ & $70 \%$ & - \\
\hline $9 \mathrm{~b}$ & $\mathrm{H}$ & $\alpha$-L-Rha & $\begin{array}{l}21 \% \text { (Scheme } 2) \\
78 \% \text { (Scheme } 3)\end{array}$ & - \\
\hline $14 a$ & $\beta$-D-Gal & $\beta$-D-Glc & $28 \%$ & - \\
\hline $14 \mathrm{~b}$ & $\alpha-\mathrm{L}-\mathrm{Ara}$ & $\beta$-D-Glc & $54 \%$ & - \\
\hline $16 a$ & $\beta$-D-Gal & $\beta-D-X y l$ & $66 \%$ & - \\
\hline $16 b$ & $\beta$-D-Xyl & $\begin{array}{c}\alpha \text {-D-Glc- }(1 \rightarrow 4)- \\
\beta \text {-D-Glc }\end{array}$ & $44 \%$ & - \\
\hline
\end{tabular}

\section{Conclusions}

In conclusion, 15 OA glycosides, including 3-monoglycosides, 28-monoglycosides and 3,28diglycosides, were easily synthesized in high yields. This method is suitable for preparing OA glycosides on a large scale and analogues for extensive biological evaluation, mechanism research and structure-activity relationship study. Moreover, this work may also provide an efficient method in preparing glycoside derivatives of other pentacyclic triterpenoid carboxylic acids, such as ursolic acid, glyrrhetinic acid and boswellic acid. Related work in this field is currently in progress and will be reported in due course.

\section{Experimental}

\section{General}

Commercial reagents were used without further treatment unless specialized. Solvents were dried and distilled prior to use in the usual way. Boiling range of petroleum ether was $60 \sim 90^{\circ} \mathrm{C}$. Analytical TLC was performed with silica gel $\mathrm{GF}_{254}$. Preparation column chromatography was performed with silica gel H. Saccharide donors (trichloroacetimidates and bromoglycosides) were prepared according to the reported methods $[16,25,26] .{ }^{1} \mathrm{H}-\mathrm{NMR}$ and ${ }^{13} \mathrm{C}-\mathrm{NMR}$ spectra were recorded on a Bruker ARX 
$300 \mathrm{MHz}$ instrument. $J$ values were given in Hz. ESI-MS were obtained on an Agilent 1100 mass spectrometer. HRMS was detected on High resolution ESI-FTICR mass spectrometry (Ion spec 7.0T).

\section{Synthesis of $O A$ 3-glycosides $\mathbf{6 a} \sim \mathbf{6 g}$}

Benzyl oleanolate (2).

A suspension of $\mathrm{OA}(5.00 \mathrm{~g}, 10.9 \mathrm{mmol}), \mathrm{BnBr}(2.10 \mathrm{~mL}, 17.5 \mathrm{mmol})$ and $\mathrm{K}_{2} \mathrm{CO}_{3}(3.00 \mathrm{~g}, 21.8$ $\mathrm{mmol})$ in THF- $\mathrm{H}_{2} \mathrm{O}(40: 1,82 \mathrm{~mL})$ was stirred overnight at $\mathrm{rt}$. The mixture was then filtered, and the filtrate was concentrated under vacuum and purified through a silica gel column chromatography (8:1, petroleum ether-EtOAc) to give $2(5.84 \mathrm{~g}, 98 \%)$ as a white amorphous solid. The physical data agreed with that previously reported [19].

\section{Oleanolic acid 3-O- $\alpha$-L-arabinopyranoside (6d)}

Benzyl ester $2(200 \mathrm{mg}, 0.366 \mathrm{mmol})$, trichloroacetimidate 3d (244 $\mathrm{mg}, 0.402 \mathrm{mmol})$ and powdered $4 \AA$ molecular sieves $(500 \mathrm{mg})$ were stirred for $30 \mathrm{~min}$ at $\mathrm{rt}$ in dry $\mathrm{CH}_{2} \mathrm{Cl}_{2}(6 \mathrm{~mL})$. A solution of TMSOTf in dry $\mathrm{CH}_{2} \mathrm{Cl}_{2}(1 \%, 0.35 \mathrm{~mL})$ was added dropwise. The mixture was stirred for 20 min followed by addition of $\mathrm{Et}_{3} \mathrm{~N}(0.10 \mathrm{~mL})$ and filtration. The filtrate was concentrated and purified by a silica gel column chromatography (8:1, petroleum ether-EtOAc) to afford benzyl oleanolate 3-O(2,3,4-tri- $O$-benzoyl- $\alpha$-L-arabinopyranoside) (4d) $(330 \mathrm{mg}, 91 \%)$ as a white amorphous solid. $R_{\mathrm{f}}=$ 0.65 (4:1, petroleum ether-EtOAc); ${ }^{1} \mathrm{H}-\mathrm{NMR}\left(\mathrm{CDCl}_{3}\right) \delta 8.09-7.26(m, 20 \mathrm{H}, \mathrm{H}-\mathrm{C}(\mathrm{Ar})), 5.75(d d, J=8.8$, 6.5, 1H, H-C(2')), $5.67\left(m, 1 \mathrm{H}, \mathrm{H}-\mathrm{C}\left(4^{\prime}\right)\right), 5.59\left(d d, J=8.9,3.5,1 \mathrm{H}, \mathrm{H}-\mathrm{C}\left(3^{\prime}\right)\right), 5.30(t, J=3.0,1 \mathrm{H}, \mathrm{H}-$ $\mathrm{C}(12)), 5.06\left(d d, J=19.9,12.6,2 \mathrm{H}, \mathrm{PhCH}_{2}\right), 4.79\left(d, J=6.6,1 \mathrm{H}, \mathrm{H}-\mathrm{C}\left(1^{\prime}\right)\right), 4.33(d d, J=12.9,3.8,1 \mathrm{H}$, H-C(5')-1), 3.87 ( $\left.m, 1 \mathrm{H}, \mathrm{H}-\mathrm{C}\left(5^{\prime}\right)-2\right), 3.16(d d, J=11.0,4.9,1 \mathrm{H}, \mathrm{H}-\mathrm{C}(3)), 2.90$ (dd, J=10.1, 3.2, 1H, H$\mathrm{C}(18)), 1.11,0.92,0.90,0.86,0.79,0.66,0.58\left(s, 7 \times 3 \mathrm{H}, \mathrm{CH}_{3}\right)$; ESI-MS: $1013.5\left[(\mathrm{M}+\mathrm{Na})^{+}\right]$.

A suspension of $\mathbf{4 d}$ (200 $\mathrm{mg}, 0.202 \mathrm{mmol})$ and 10\% Pd-C (32 mg) in EtOAc (8 mL) was refluxed and bubbled up with $\mathrm{H}_{2}(20 \mathrm{~mL} / \mathrm{min})$ for $3 \mathrm{~h}$. The mixture was then filtered and the filtrate was concentrated to dryness to afford oleanolic acid 3-O-(2,3,4-tri- $O$-benzoyl- $\alpha$-L-arabinopyranoside) (5d) $(180 \mathrm{mg}, 99 \%)$ as a white amorphous solid. $R_{\mathrm{f}}=0.36\left(3: 1\right.$, petroleum ether-EtOAc); ${ }^{1} \mathrm{H}-\mathrm{NMR}\left(\mathrm{CDCl}_{3}\right)$ $\delta$ 8.07-7.18 ( $m, 15 \mathrm{H}, \mathrm{H}-\mathrm{C}(\mathrm{Ar})), 5.77\left(d d, 1 \mathrm{H}, J=8.8,6.4, \mathrm{H}-\mathrm{C}\left(2^{\prime}\right)\right), 5.67\left(m, 1 \mathrm{H}, \mathrm{H}-\mathrm{C}\left(4^{\prime}\right)\right), 5.59$ (dd, $\left.1 \mathrm{H}, J=8.8,3.5, \mathrm{H}-\mathrm{C}\left(3^{\prime}\right)\right), 5.26(b r s, 1 \mathrm{H}, \mathrm{H}-\mathrm{C}(12)), 4.78\left(d, 1 \mathrm{H}, J=6.3, \mathrm{H}-\mathrm{C}\left(1^{\prime}\right)\right), 4.33$ (dd, $1 \mathrm{H}, J=13.0$, 3.9, H-C(5')-1), 3.87 (dd, 1H, J=13.0, 1.9, H-C(5')-2), 3.16 (dd, 1H, J=11.1, 4.8, H-C(3)), 2.80 (dd, $1 \mathrm{H}, J=9.9,2.6, \mathrm{H}-\mathrm{C}(18)), 1.10,0.92,0.90,0.87,0.77,0.70,0.62\left(s, 7 \times 3 \mathrm{H}, \mathrm{CH}_{3}\right)$; ESI-MS: 918.8 $\left[\left(\mathrm{M}+\mathrm{NH}_{4}\right)^{+}\right]$.

Compound 5d (84 mg, $0.093 \mathrm{mmol}$ ) was suspended in dry $\mathrm{MeOH}(5 \mathrm{~mL})$, to which a freshly prepared solution of $\mathrm{NaOMe}$ in $\mathrm{MeOH}(1.0 \mathrm{M}, 0.40 \mathrm{~mL})$ was added. The mixture was stirred at $\mathrm{rt}$ overnight and then neutralized with Dowex $\mathrm{H}^{+}$resin to $\mathrm{pH} 7$ and filtered. The filtrate was concentrated and purified with a silica gel column chromatography $\left(9: 1, \mathrm{CHCl}_{3}-\mathrm{MeOH}\right)$ to give oleanolic acid 3-O$\alpha$-L-arabinopyranoside (6d) $(47 \mathrm{mg}, 85 \%)$ as a white powder. $R_{\mathrm{f}}=0.56\left(5: 1, \mathrm{CHCl}_{3}-\mathrm{MeOH}\right) ;{ }^{1} \mathrm{H}-\mathrm{NMR}$ (pyridine- $\left.d_{5}\right) \delta 5.47(b r s, 1 \mathrm{H}, \mathrm{H}-\mathrm{C}(12)), 4.77\left(d, 1 \mathrm{H}, J=7.0, \mathrm{H}-\mathrm{C}\left(1^{\prime}\right)\right), 4.43(t, 1 \mathrm{H}, J=8.7), 4.31(m$, $2 \mathrm{H}), 4.16(d d, 1 \mathrm{H}, J=8.7,3.1), 3.83(m, 1 \mathrm{H}), 3.37-3.27$ ( $m, 2 \mathrm{H}, \mathrm{H}-\mathrm{C}(3), \mathrm{H}-\mathrm{C}(18)), 1.29,1.27,1.00$, 0.99, 0.94, 0.93, $0.83\left(s, 7 \times 3 \mathrm{H}, \mathrm{CH}_{3}\right) ;{ }^{13} \mathrm{C}-\mathrm{NMR}$ (pyridine- $\left.d_{5}\right) \delta 180.2,144.8,122.6,107.6,88.7,74.7$, 
73.0, 69.6, 66.9, 55.9, 48.1, 46.7, 46.5, 42.2, 42.0, 39.8, 39.6, 38.8, 37.0, 34.2, 33.3, 33.2, 33.2, 31.0, 28.2, 28.2, 26.7, 26.2, 23.8, 23.8, 23.7, 18.5, 17.4, 16.9, 15.5; ESI-MS: $611.5\left[(\mathrm{M}+\mathrm{Na})^{+}\right], 627.3$ $\left[(\mathrm{M}+\mathrm{K})^{+}\right]$. HRMS: $m / z 587.3962[\mathrm{M}-\mathrm{H}]^{-}\left(\left[\mathrm{C}_{35} \mathrm{H}_{55} \mathrm{O}_{7}\right]=587.3953\right)$.

Compounds $6 \mathbf{a} \sim \mathbf{6 c}$ and $\mathbf{6 e} \sim \mathbf{6 g}$ were prepared according to the same procedure described for $\mathbf{6 d}$.

Oleanolic acid 3-O- $\beta$-D-glucopyranoside (6a). $R_{\mathrm{f}}=0.39$ (5:1, $\left.\mathrm{CHCl}_{3}-\mathrm{MeOH}\right) ;{ }^{1} \mathrm{H}-\mathrm{NMR}$ (pyridine- $d_{5}$ ) $\delta 5.44$ (br s, 1H, H-C(12)), $4.93\left(d, 1 \mathrm{H}, J=7.7, \mathrm{H}-\mathrm{C}\left(1^{\prime}\right)\right), 4.57$ (br d, 1H, J=11.3), $4.43(m, 1 \mathrm{H}), 4.25$ $(m, 2 \mathrm{H}), 4.01(m, 2 \mathrm{H}), 3.46(d d, 1 \mathrm{H}, J=12.5,2.2, \mathrm{H}-\mathrm{C}(18)), 3.37$ (dd, 1H, $J=8.0,2.4, \mathrm{H}-\mathrm{C}(3)), 1.31$, $1.30,1.01,0.98,0.93,0.91,0.80\left(s, 7 \times 3 \mathrm{H}, \mathrm{CH}_{3}\right) ;{ }^{13} \mathrm{C}-\mathrm{NMR}$ (pyridine- $\left.d_{5}\right) \delta 180.2,145.4,122.4,107.0$, 88.8, 78.8, 78.5, 78.4, 71.8, 63.0, 55.8, 48.0, 46.8, 46.7, 42.2, 42.1, 39.7, 39.5, 38.7, 37.0, 34.4, 33.4, $33.3,33.0,31.1,30.0,29.9,28.3,26.2,23.9,23.9,23.8,18.5,17.5,17.1,15.5$; ESI-MS: 641.4 $\left[(\mathrm{M}+\mathrm{Na})^{+}\right], 653.4\left[(\mathrm{M}+\mathrm{Cl})^{-}\right]$. HRMS: $m / z 617.4071[\mathrm{M}-\mathrm{H}]^{-}\left(\left[\mathrm{C}_{36} \mathrm{H}_{57} \mathrm{O}_{8}\right]=617.4059\right)$.

Oleanolic acid 3-O- $\beta$-D-galactopyranoside $(\mathbf{6 b}) . R_{\mathrm{f}}=0.40\left(5: 1, \mathrm{CHCl}_{3}-\mathrm{MeOH}\right) ;{ }^{1} \mathrm{H}-\mathrm{NMR}\left(\mathrm{DMSO}-d_{6}\right)$ $\delta 12.07(s, 1 \mathrm{H}, \mathrm{COOH}), 5.16(b r s, 1 \mathrm{H}, \mathrm{H}-\mathrm{C}(12)), 4.75,4.64,4.52,4.33(b r s, 4 \times 1 \mathrm{H}, \mathrm{OH}), 4.11(d, 1 \mathrm{H}$, $\left.J=6.4, \mathrm{H}-\mathrm{C}\left(1^{\prime}\right)\right), 3.62(b r s, 1 \mathrm{H}), 3.52(m, 1 \mathrm{H}), 3.43(\mathrm{~m}, 1 \mathrm{H}), 3.29-3.25(m, 3 \mathrm{H}), 3.03(b r d, J=7.8, \mathrm{H}-$ $\mathrm{C}(3)), 2.74$ (br d, 1H, J=10.6, H-C(18)), $1.10\left(s, 3 \mathrm{H}, \mathrm{CH}_{3}\right), 0.98\left(s, 3 \mathrm{H}, \mathrm{CH}_{3}\right), 0.88\left(s, 9 \mathrm{H}, 3 \times \mathrm{CH}_{3}\right)$, $0.76\left(s, 3 \mathrm{H}, \mathrm{CH}_{3}\right), 0.72\left(s, 3 \mathrm{H}, \mathrm{CH}_{3}\right) ;{ }^{13} \mathrm{C}-\mathrm{NMR}$ (DMSO- $\left.d_{6}\right) \delta 178.7,143.9,121.6,106.2,88.0,75.0$, 73.7, 71.2, 68.2, 60.4, 55.1, 47.2, 45.8, 45.6, 41.4, 40.9, 38.7, 38.3, 38.2, 36.4, 33.4, 32.9, 32.5, 32.2, 30.5, 27.8, 27.3, 25.7, 25.6, 23.5, 23.0, 22.7, 17.9, 16.9, 16.6, 15.2; ESI-MS: $641.4\left[(\mathrm{M}+\mathrm{Na})^{+}\right]$. HRMS: $m / z 617.4062[\mathrm{M}-\mathrm{H}]^{-}\left(\left[\mathrm{C}_{36} \mathrm{H}_{57} \mathrm{O}_{8}\right]=617.4059\right)$.

Oleanolic acid 3-O- $\beta$-D-xylopyranoside $(6 \mathbf{6 c}) . R_{\mathrm{f}}=0.69\left(5: 1, \mathrm{CHCl}_{3}-\mathrm{MeOH}\right) ;{ }^{1} \mathrm{H}-\mathrm{NMR}$ (pyridine- $\left.d_{5}\right) \delta$ 5.45 (br s, 1H, H-C(12)), $4.84\left(d, 1 \mathrm{H}, J=7.5, \mathrm{H}-\mathrm{C}\left(1^{\prime}\right)\right), 4.39$ (dd, 1H, J=11.2, 4.9), 4.25-4.15 (m, 2H), $4.03(t, 1 \mathrm{H}, J=7.8), 3.79(t, 1 \mathrm{H}, J=10.4), 3.44$ ( $b r d, 1 \mathrm{H}, J=10.3, \mathrm{H}-\mathrm{C}(18)), 3.35$ (dd, 1H, $J=11.2,3.8$, $\mathrm{H}-\mathrm{C}(3)), 1.31,1.30,1.02,0.98,0.95,0.94,0.85\left(s, 7 \times 3 \mathrm{H}, \mathrm{CH}_{3}\right) ;{ }^{13} \mathrm{C}-\mathrm{NMR}$ (pyridine- $\left.d_{5}\right) \delta 180.4$, 144.9, 122.5, 107.7, 88.6, 78.7, 75.6, 71.3, 67.2, 55.9, 48.1, 46.7, 46.5, 42.2, 42.0, 39.7, 39.6, 38.8, 37.0, 34.3, 33.3, 33.3, 33.2, 31.0, 28.3, 28.2, 26.8, 26.2, 23.8, 23.8, 23.7, 18.5, 17.4, 17.0, 15.5; ESIMS: $611.5\left[(\mathrm{M}+\mathrm{Na})^{+}\right]$. HRMS: $m / z 587.3953[\mathrm{M}-\mathrm{H}]^{-}\left(\left[\mathrm{C}_{35} \mathrm{H}_{55} \mathrm{O}_{7}\right]=587.3953\right)$.

Oleanolic acid 3-O- $\beta$-D-galactopyranosyl-(1 $\rightarrow 4)$ - $\beta$-D-glucopyranoside $(\mathbf{6 e}) . R_{\mathrm{f}}=0.16\left(4: 1, \mathrm{CHCl}_{3^{-}}\right.$ $\mathrm{MeOH}$ ); ${ }^{1} \mathrm{H}-\mathrm{NMR}$ (DMSO- $\left.d_{6}\right) 5.16$ (br s, 1H, H-C(12)), 4.23-4.21 (m, 2H, H-C(1'), H-C(1")), 3.733.69 (br d, 1H, J=11.0), 3.61-3.44 (m, 5H), 3.33-3.25 (m, 5H), 3.04-3.01 (m, 2H, H-C(2'), H-C(3)), $2.73(d d, 1 \mathrm{H}, J=12.8,3.4, \mathrm{H}-\mathrm{C}(18)), 1.09\left(s, 3 \mathrm{H}, \mathrm{CH}_{3}\right), 0.98\left(s, 3 \mathrm{H}, \mathrm{CH}_{3}\right), 0.87\left(s, 9 \mathrm{H}, 3 \times \mathrm{CH}_{3}\right), 0.75$ $\left(s, 3 \mathrm{H}, \mathrm{CH}_{3}\right), 0.71\left(s, 3 \mathrm{H}, \mathrm{CH}_{3}\right) ;{ }^{13} \mathrm{C}-\mathrm{NMR}$ (DMSO- $\left.d_{6}\right) \delta 178.8,144.0,121.7,105.3,104.1,88.3,81.3$, 75.6, 75.2, 74.7, 73.7, 73.3, 70.7, 68.2, 60.8, 60.5, 55.2, 47.3, 45.9, 45.6, 41.5, 40.9, 38.8, 38.7, 38.3, $36.5,33.5,33.0,32.6,32.3,30.6,27.8,27.4,25.8,25.8,23.5,23.1,22.8,18.0,17.0,16.7$, 15.3; ESIMS: $781.0\left[(\mathrm{M}+\mathrm{H})^{+}\right], 798.0\left[\left(\mathrm{M}+\mathrm{NH}_{4}\right)^{+}\right]$. HRMS: $m / z 779.4580[\mathrm{M}-\mathrm{H}]^{-}\left(\left[\mathrm{C}_{42} \mathrm{H}_{67} \mathrm{O}_{13}\right]=779.4587\right)$.

Oleanolic acid 3-O- $\alpha$-D-glucopyranosyl-(1 $\rightarrow 4)$ - $\beta$-D-glucopyranoside (6f). $R_{\mathrm{f}}=0.13\left(4: 1, \mathrm{CHCl}_{3^{-}}\right.$ $\mathrm{MeOH}$ ); ${ }^{1} \mathrm{H}-\mathrm{NMR}$ (pyridine- $\left.d_{5}\right) \delta 5.78(d, 1 \mathrm{H}, J=3.5, \mathrm{H}-\mathrm{C}(1 ")), 5.50$ (br s, 1H, H-C(12)), 5.32 (br $d$, 
$1 \mathrm{H}, J=11.5), 5.08(d d, 1 \mathrm{H}, J=9.5,5.6), 4.82\left(d, 1 \mathrm{H}, J=7.7, \mathrm{H}-\mathrm{C}\left(1^{\prime}\right)\right), 4.63-4.50(m, 3 \mathrm{H}), 4.43-4.31(m$, 2H), 4.24-4.04 (m, 5H), 3.28 (m, 2H, H-C(3), H-C(18)), 1.29, 1.25, 1.01, 0.96, 0.95, 0.90, $0.76(s$, $7 \times 3 \mathrm{H}, \mathrm{CH}_{3}$ ) ${ }^{13} \mathrm{C}-\mathrm{NMR}$ (pyridine- $\left.d_{5}\right) \delta 180.2,144.9,122.6,106.8,103.8,89.6,82.9,77.9,75.6,75.4$, 74.9, 74.4, 73.3, 71.6, 65.1, 62.5, 55.9, 48.0, 46.7, 46.5, 42.2, 42.0, 39.7, 39.4, 38.5, 36.9, 34.3, 33.3, 33.2, 33.2, 31.0, 28.3, 28.2, 26.5, 26.3, 23.8, 23.8, 23.7, 18.5, 17.4, 16.9, 15.4; ESI-MS: 779.2 [(M-H) ], $803.4\left[(\mathrm{M}+\mathrm{Na})^{+}\right]$. HRMS: $m / z 779.4584[\mathrm{M}-\mathrm{H}]^{-}\left(\left[\mathrm{C}_{42} \mathrm{H}_{67} \mathrm{O}_{13}\right]=779.4587\right)$.

Oleanolic acid 3-O- $\alpha$-L-rhamnopyranoside (6g). $R_{\mathrm{f}}=0.80\left(5: 1, \mathrm{CHCl}_{3}-\mathrm{MeOH}\right) ;{ }^{1} \mathrm{H}-\mathrm{NMR}\left(\mathrm{DMSO}-d_{6}\right)$ $\delta 12.05$ ( $s, 1 \mathrm{H}, \mathrm{COOH}), 5.16$ (br s, 1H, H-C(12)), 4.70 ( $\left.m, 2 \mathrm{H}, \mathrm{H}-\mathrm{C}\left(1^{\prime}\right), \mathrm{OH}\right), 4.58(s, 1 \mathrm{H}, \mathrm{OH}), 4.52$ $(d, 1 \mathrm{H}, J=5.8, \mathrm{OH}), 3.62(b r s, 1 \mathrm{H}), 3.49(m, 1 \mathrm{H}), 3.40(m, 1 \mathrm{H}), 3.17(m, 1 \mathrm{H}), 3.01(d d, 1 \mathrm{H}, J=9.7$, 3.0, H-C(3)), 2.74 (br d, 1H, J=10.8, H-C(18)), $1.10\left(s, 3 \mathrm{H}, \mathrm{CH}_{3}\right), 1.09\left(s, 3 \mathrm{H}, \mathrm{CH}_{3}\right), 0.87(s, 9 \mathrm{H}$, $\left.3 \times \mathrm{CH}_{3}\right), 0.72\left(s, 6 \mathrm{H}, 2 \times \mathrm{CH}_{3}\right) ;{ }^{13} \mathrm{C}-\mathrm{NMR}\left(\mathrm{DMSO}-d_{6}\right) \delta 178.7,143.9,121.6,103.0,87.6,72.2,70.8$, 70.8, 68.6, 54.7, 47.1, 45.8, 45.5, 41.4, 40.9, 38.8, 38.6, 37.9, 36.4, 33.4, 32.9, 32.4, 32.2, 30.5, 28.0, $27.3,25.7,25.0,23.5,23.0,22.7,17.9,17.8,16.9,16.5,15.2$; ESI-MS: 637.6 [(M+Cl) $\left.{ }^{-}\right]$. HRMS: $\mathrm{m} / \mathrm{z}$ $601.4104[\mathrm{M}-\mathrm{H}]^{-}\left(\left[\mathrm{C}_{36} \mathrm{H}_{57} \mathrm{O}_{7}\right]=601.4110\right)$.

Synthesis of OA 28-glycosides 9a 9b, approach 1: $\beta$-D-galactopyranosyl oleanolate (9a)

$\mathrm{Ac}_{2} \mathrm{O}(18.9 \mathrm{~mL})$ was added dropwise to a solution of OA $(9.14 \mathrm{~g}, 20 \mathrm{mmol})$ in dry pyridine (38 $\mathrm{mL})$ at $0^{\circ} \mathrm{C}$ under stirring. To the mixture was added DMAP $(244 \mathrm{mg}, 2 \mathrm{mmol})$ and the mixture was allowed to warm up to rt and stirred overnight. Water $(15 \mathrm{~mL})$ was added to quench the reaction. The mixture was then concentrated in vacuum and the residue was dissolved in $\mathrm{CH}_{2} \mathrm{Cl}_{2}(150 \mathrm{~mL})$ and washed with $5 \% \mathrm{HCl}$, saturated $\mathrm{NaHCO}_{3}$ and brine in sequence. The solution was dried over $\mathrm{Na}_{2} \mathrm{SO}_{4}$. Recrystallization with $\mathrm{MeOH}-\mathrm{CH}_{2} \mathrm{Cl}_{2}$ gave oleanolic acid 3-acetate (7) (7.66 g, 77\%) as a white powder. $R_{\mathrm{f}}=0.78\left(3: 1\right.$, petroleum ether-EtOAc); ${ }^{1} \mathrm{H}-\mathrm{NMR}\left(\mathrm{CDCl}_{3}\right) \delta 5.27(\mathrm{br} \mathrm{s}, 1 \mathrm{H}, \mathrm{H}-\mathrm{C}(12)), 4.49$ $(d d, 1 \mathrm{H}, J=8.2,7.5, \mathrm{H}-\mathrm{C}(3)), 2.82$ ( $b r d, 1 \mathrm{H}, J=10.0, \mathrm{H}-\mathrm{C}(18)), 2.05$ (s, 3H, Ac), 1.12, 0.94, 0.93, 0.90, 0.86, 0.85, $0.74\left(s, 7 \times 3 \mathrm{H}, \mathrm{CH}_{3}\right)$; ESI-MS: $521.8\left[(\mathrm{M}+\mathrm{Na})^{+}\right]$.

A suspension of 7 (250 mg, $0.501 \mathrm{mmol}), 3 \mathbf{b}(480 \mathrm{mg}, 0.648 \mathrm{mmol})$ and powdered $4 \AA$ molecular sieves $(800 \mathrm{mg})$ in dry $\mathrm{CH}_{2} \mathrm{Cl}_{2}(8 \mathrm{~mL})$ were stirred for $30 \mathrm{~min}$ at $0^{\circ} \mathrm{C}$. TMSOTf $(40 \mu \mathrm{L})$ was added and the mixture was stirred for $20 \mathrm{~min}$ before $\mathrm{Et}_{3} \mathrm{~N}(0.15 \mathrm{~mL})$ was added to quench the reaction. The mixture was then filtered and the filtrate was concentrated and purified by a silica gel column chromatography (7:1, petroleum ether-EtOAc) to afford 3-O-acetyloleanolic acid 2,3,4,6-tetra- $O$ benzoyl- $\beta$-D-galactopyranosyl ester (8a) $(391 \mathrm{mg}, 72 \%)$ as a white amorphous solid. $R_{\mathrm{f}}=0.70(4: 1$, petroleum ether-EtOAc); ${ }^{1} \mathrm{H}-\mathrm{NMR}\left(\mathrm{CDCl}_{3}\right) \delta$ 8.10-7.21 ( $\left.m, 20 \mathrm{H}, \mathrm{H}-\mathrm{C}(\mathrm{Ar})\right), 6.02(d, 1 \mathrm{H}, J=3.3), 5.93$ $(m, 2 \mathrm{H}), 5.73(m, 1 \mathrm{H}), 5.31$ (br s, 1H, H-C(12)), 4.62 (dd, 1H, J=10.6, 6.1), 4.46-4.33 (m, 3H), 2.82 $(d d, 1 \mathrm{H}, J=11.8,2.1$, H-C(18)), $2.04(s, 3 \mathrm{H}, \mathrm{Ac}), 0.97,0.88,0.85,0.83,0.82,0.81,0.49(s, 7 \times 3 \mathrm{H}$, $\left.\mathrm{CH}_{3}\right)$; ESI-MS: $1099.8\left[(\mathrm{M}+\mathrm{Na})^{+}\right]$.

8a (94 mg, $0.087 \mathrm{mmol})$ was dissolved in dry $\mathrm{MeOH}(8 \mathrm{ml})$, to which a freshly prepared solution of $\mathrm{NaOMe}$ in $\mathrm{MeOH}(1.0 \mathrm{M}, 1.60 \mathrm{~mL})$ was added. The mixture was stirred at $\mathrm{rt}$ for $12 \mathrm{~h}$ and then neutralized with Dowex $\mathrm{H}^{+}$resin to $\mathrm{pH} 7$ and filtered. The filtrate was concentrated and purified with a silica gel column chromatography $\left(10: 1, \mathrm{CHCl}_{3}-\mathrm{MeOH}\right)$ to give $\beta$-D-galactopyranosyl oleanolate (9a) $(17 \mathrm{mg}, 32 \%)$ as a white powder. $R_{\mathrm{f}}=0.43\left(8: 1, \mathrm{CHCl}_{3}-\mathrm{MeOH}\right) ;{ }^{1} \mathrm{H}-\mathrm{NMR}$ (pyridine- $\left.d_{5}\right) \delta 6.27(d, 1 \mathrm{H}$, 
$\left.J=8.0, \mathrm{H}-\mathrm{C}\left(1^{\prime}\right)\right), 5.44$ (br s, 1H, H-C(12)), 4.69-4.64 (m, 2H), $4.48(m, 1 \mathrm{H}), 4.40(m, 1 \mathrm{H}), 4.21(m, 2 \mathrm{H})$, $3.43(d d, 1 \mathrm{H}, J=9.5,5.6, \mathrm{H}-\mathrm{C}(3)), 3.19$ ( $b r d, 1 \mathrm{H}, J=11.1, \mathrm{H}-\mathrm{C}(18)), 1.21,1.21,1.14,1.02,0.91,0.88$, $0.86\left(s, 7 \times 3 \mathrm{H}, \mathrm{CH}_{3}\right) ;{ }^{13} \mathrm{C}-\mathrm{NMR}$ (pyridine- $\left.d_{5}\right) \delta 176.0,143.7,122.4,95.8,77.6,77.3,75.2,71.0,69.6$, 61.4, 55.3, 47.7, 46.5, 45.7, 41.6, 41.3, 39.4, 38.9, 38.5, 36.9, 33.5, 32.7, 32.7, 32.0, 30.3, 28.3, 27.8, 27.6, 25.6, 23.4, 23.2, 22.8, 18.3, 17.0, 16.1, 15.2; ESI-MS: $641.4\left[(\mathrm{M}+\mathrm{Na})^{+}\right]$. HRMS: $m / z 617.4058$ $[\mathrm{M}-\mathrm{H}]^{-}\left(\left[\mathrm{C}_{36} \mathrm{H}_{57} \mathrm{O}_{8}\right]=617.4059\right)$.

\section{$\alpha$-L-Rhamnopyranosyl oleanolate (9b)}

This compound was prepared according to the same procedure described for 9a. $R_{\mathrm{f}}=0.60(8: 1$, $\mathrm{CHCl}_{3}-\mathrm{MeOH}$ ); ${ }^{1} \mathrm{H}-\mathrm{NMR}$ (pyridine- $\left.d_{5}\right) \delta 6.79(s, 1 \mathrm{H}, J=1.4$, H-C(1')), 5.43 (br s, 1H, H-C(12)), 4.58 $(m, 1 \mathrm{H}), 4.51(d d, 1 \mathrm{H}, J=8.6,3.1), 4.41-4.36(m, 2 \mathrm{H}), 3.44$ (dd, 1H, J=9.4, 6.1, H-C(3)), 3.14 (br $d$, $1 \mathrm{H}, J=10.2$, H-C(18)), $1.70\left(d, 3 \mathrm{H}, J=5.4, \mathrm{H}-\mathrm{C}\left(6^{\prime}\right)\right), 1.23,1.20,1.04,1.02,0.90,0.90,0.86(s, 7 \times 3 \mathrm{H}$, $\mathrm{CH}_{3}$ ); ${ }^{13} \mathrm{C}-\mathrm{NMR}$ (pyridine- $d_{5}$ ) $\delta 175.9,143.9,122.6,95.4,78.1,73.4,72.9,72.6,71.6,55.8,48.0,47.4$, 46.0, 42.2, 42.1, 39.8, 39.4, 39.0, 37.4, 33.9, 33.3, 33.1, 33.0, 30.9, 28.8, 28.1, 28.0, 26.0, 23.9, 23.6, 23.3, 18.8, 18.8, 17.7, 16.6, 15.6; ESI-MS: 637.6 [(M+Cl) $\left.{ }^{-}\right]$. HRMS: $m / z 601.4105[\mathrm{M}-\mathrm{H}]^{-}\left(\left[\mathrm{C}_{36} \mathrm{H}_{57} \mathrm{O}_{7}\right]\right.$ $=601.4110)$.

Synthesis of OA 28-glycosides 12a 12d, approach 2: $\beta$-D-xylopyranosyl oleanolate (12b)

OA (100 mg, $0.219 \mathrm{mmol}$ ), benzoylbromoglycoside $10 \mathrm{~b}$ (138 mg, $0.263 \mathrm{mmol})$, and $\mathrm{Bu}_{4} \mathrm{NBr}(4$ $\mathrm{mg}$ ) were dissolved in $\mathrm{CHCl}_{3}(4.0 \mathrm{~mL}) ; \mathrm{K}_{2} \mathrm{CO}_{3}(152 \mathrm{mg})$ was dissolved in water $(1.5 \mathrm{~mL})$. The two solutions were mixed together and stirred vigorously at $50^{\circ} \mathrm{C}$ for $2.5 \mathrm{~h}$. The organic layer was separated and diluted with $\mathrm{CHCl}_{3}(10 \mathrm{~mL})$ and washed by water $(10 \mathrm{~mL} \times 2)$ and concentrated to give a brown residue, which was subjected to a column chromatography $(2: 1$, petroleum ether-EtOAc) to afford oleanolic acid 2,3,4-tri- $O$-benzoyl- $\beta$-D-xylopyranosyl ester (11b) (183 $\mathrm{mg}, 92 \%)$ as a white foam. $R_{\mathrm{f}}=0.32$ (3:1, petroleum-EtOAc); ${ }^{1} \mathrm{H}-\mathrm{NMR}\left(\mathrm{CDCl}_{3}\right) \delta 8.01-7.32(\mathrm{~m}, 15 \mathrm{H}, \mathrm{H}-\mathrm{C}(\mathrm{Ar})), 5.89(\mathrm{~m}$, $2 \mathrm{H}), 5.63(d d, 1 \mathrm{H}, J=8.8,7.4), 5.40(t d, 1 \mathrm{H}, J=8.8,5.2), 5.28(t, 1 \mathrm{H}, J=3.3, \mathrm{H}-\mathrm{C}(12)), 4.42(d d, 1 \mathrm{H}$, $J=11.9,5.1), 3.74(d d, 1 \mathrm{H}, J=11.9,8.9), 3.16$ (dd, 1H, $J=11.1,4.2, \mathrm{H}-\mathrm{C}(3)), 2.81$ ( $d d, 1 \mathrm{H}, J=13.4,3.8$, H-C(18)), 0.97, 0.95, 0.87, 0.85, 0.84, 0.76, $0.49\left(s, 7 \times 3 \mathrm{H}, \mathrm{CH}_{3}\right)$; ESI-MS: $918.8\left[\left(\mathrm{M}+\mathrm{NH}_{4}\right)^{+}\right]$.

Compound 11b (190 mg, $0.211 \mathrm{mmol})$ was dissolved in dry $\mathrm{CH}_{2} \mathrm{Cl}_{2}-\mathrm{MeOH}(1: 2,6 \mathrm{~mL})$ and a freshly prepared solution of $\mathrm{NaOMe}$ in $\mathrm{MeOH}(1.0 \mathrm{M}, 0.50 \mathrm{~mL})$ was added. The mixture was stirred at $\mathrm{rt}$ for $2 \mathrm{~h}$ and then neutralized with Dowex $\mathrm{H}^{+}$resin to $\mathrm{pH} 7$ and filtered. The filtrate was concentrated and purified with a silica gel column chromatography $\left(12: 1, \mathrm{CHCl}_{3}-\mathrm{MeOH}\right)$ to give $\beta$-D-xylopyranosyl oleanolate (12b) $(100 \mathrm{mg}, 80 \%)$ as a white powder. $R_{\mathrm{f}}=0.50\left(10: 1, \mathrm{CHCl}_{3}-\mathrm{MeOH}\right) ;{ }^{1} \mathrm{H}-\mathrm{NMR}$ (pyridine- $\left.d_{5}\right) \delta 6.24\left(d, 1 \mathrm{H}, J=6.7, \mathrm{H}-\mathrm{C}\left(1^{\prime}\right)\right), 5.46(b r s, 1 \mathrm{H}, \mathrm{H}-\mathrm{C}(12)), 4.38(d d, 1 \mathrm{H}, J=11.7,4.4), 4.22$ $(m, 3 \mathrm{H}), 3.83(m, 1 \mathrm{H}), 3.43(\mathrm{~m}, 1 \mathrm{H}, \mathrm{H}-\mathrm{C}(3)), 3.25(d d, 1 \mathrm{H}, J=11.8,2.6, \mathrm{H}-\mathrm{C}(18)), 1.23(s, 6 \mathrm{H}$, $\left.2 \times \mathrm{CH}_{3}\right), 1.10,1.02,0.92\left(s, 3 \times 3 \mathrm{H}, \mathrm{CH}_{3}\right), 0.90\left(s, 6 \mathrm{H}, 2 \times \mathrm{CH}_{3}\right) ;{ }^{13} \mathrm{C}-\mathrm{NMR}$ (pyridine- $\left.d_{5}\right) \delta 176.6,144.1$, 123.0, 96.3, 78.4, 78.1, 73.7, 70.9, 67.8, 55.8, 48.1, 47.2, 46.2, 42.2, 41.7, 39.9, 39.4, 38.9, 37.4, 34.0, $33.2,33.1,32.8,30.8,28.8,28.3,28.1,26.1,23.8,23.6,23.4,18.8,17.5,16.6,15.6$; ESI-MS: 611.5 $\left[(\mathrm{M}+\mathrm{Na})^{+}\right]$. HRMS: $m / z 587.3950[\mathrm{M}-\mathrm{H}]^{-}\left(\left[\mathrm{C}_{35} \mathrm{H}_{55} \mathrm{O}_{7}\right]=587.3953\right)$.

$\beta$-D-Galactopyranosyl oleanolate (9a), $\alpha$-D-glucopyranosyl-( $1 \rightarrow 4)$ - $\beta$-D-glucopyranosyl oleanolate (12c) and $\alpha$-L-rhamnopyranosyl oleanolate $(\mathbf{9 b})$ were also prepared according to the same procedure 
described for $12 \mathrm{~b}$. The analytical data of $9 \mathbf{a}$ and $9 \mathbf{b}$ were identical to that of $9 \mathbf{a}$ and $\mathbf{9 b}$ prepared by approach 1.

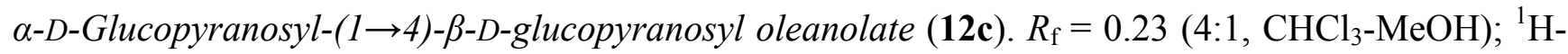
NMR (pyridine- $\left.d_{5}\right) \delta 6.23\left(d, 1 \mathrm{H}, J=8.0, \mathrm{H}-\mathrm{C}\left(1^{\prime}\right)\right), 5.95\left(d, 1 \mathrm{H}, J=3.2, \mathrm{H}-\mathrm{C}\left(1^{\prime \prime}\right)\right), 5.44$ (br s, 1H, H$\mathrm{C}(12)), 4.61-4.53(m, 3 \mathrm{H}), 4.49(m, 2 \mathrm{H}), 4.41-4.32(m, 3 \mathrm{H}), 4.20-4.15(m, 3 \mathrm{H}), 3.87$ (br d, 1H, J=8.8), $3.43(m, 1 \mathrm{H}, \mathrm{H}-\mathrm{C}(3)), 3.19(b r d, 1 \mathrm{H}, J=10.4, \mathrm{H}-\mathrm{C}(18)), 1.22\left(s, 6 \mathrm{H}, 2 \times \mathrm{CH}_{3}\right), 1.10,1.02(s, 2 \times 3 \mathrm{H}$, $\left.\mathrm{CH}_{3}\right), 0.90\left(s, 6 \mathrm{H}, 2 \times \mathrm{CH}_{3}\right), 0.88\left(s, 3 \mathrm{H}, \mathrm{CH}_{3}\right) ;{ }^{13} \mathrm{C}-\mathrm{NMR}$ (pyridine- $\left.d_{5}\right) \delta 176.4,144.1,123.2,103.1$, 95.5, 80.6, 78.3, 78.1, 77.6, 75.5, 75.4, 74.4, 73.6, 71.9, 62.7, 61.4, 55.8, 48.2, 47.0, 46.2, 42.2, 41.8, 39.9, 39.4, 39.0, 37.4, 34.0, 33.2, 33.2, 32.6, 30.8, 28.8, 28.3, 28.1, 26.1, 23.9, 23.7, 23.4, 18.8, 17.5, 16.6, 15.7; ESI-MS: $779.2\left[(\mathrm{M}-\mathrm{H})^{-}\right]$. HRMS: $m / z 779.4585[\mathrm{M}-\mathrm{H}]^{-}\left(\left[\mathrm{C}_{42} \mathrm{H}_{67} \mathrm{O}_{13}\right]=779.4587\right)$.

Synthesis of $O A$ 3,28-diglycosides 14a 14b, approach 1: $\beta$-D-glucopyranosyl oleanolate 3-O- $\alpha-L$ arabinopyranoside $\mathbf{( 1 4 b )}$.

A mixture of intermediate $5 \mathbf{d}(290 \mathrm{mg}, 0.322 \mathrm{mmol})$, trichloroacetimidate 3a (286 $\mathrm{mg}, 0.386$ mmol) and powdered $4 \AA$ molecular sieves $(1 \mathrm{~g})$ in dry $\mathrm{CH}_{2} \mathrm{Cl}_{2}(8 \mathrm{~mL})$ were stirred for $30 \mathrm{~min}$ at $0^{\circ} \mathrm{C}$. A solution of TMSOTf in dry $\mathrm{CH}_{2} \mathrm{Cl}_{2}(5 \%, 0.50 \mathrm{~mL})$ was added dropwise and the mixture was warmed to $\mathrm{rt}$ and stirred for $45 \mathrm{~min}$ before quenching the reaction with $\mathrm{Et}_{3} \mathrm{~N}(0.10 \mathrm{~mL})$. The mixture was then filtered and the filtrate was concentrated and purified by a silica gel column chromatography (3:1, petroleum ether-EtOAc) to afford $2,3,4,6$-tetra- $O$-benzoyl- $\beta$-D-glucopyranosyl oleanolate $3-O$ (2,3,4-tri- $O$-benzoyl- $\alpha$-L-arabinopyranoside) (13b) $(438 \mathrm{mg}, 92 \%)$ as a white powder. $R_{\mathrm{f}}=0.22(3: 1$, petroleum ether-EtOAc); ${ }^{1} \mathrm{H}-\mathrm{NMR} \delta$ : $\left(\mathrm{CDCl}_{3}\right) \delta$ 8.08-7.25 (m, 35H, H-C(Ar)), $5.96(d d, 1 \mathrm{H}, J=19.2$, 9.7), $5.93(d, 1 \mathrm{H}, J=8.3$, H-C(1")), 5.78-5.66 ( $m, 4 \mathrm{H}), 5.58(d d, 1 \mathrm{H}, J=8.9,3.4), 5.27$ (br s, 1H, H$\mathrm{C}(12)), 4.75\left(d, 1 \mathrm{H}, J=6.5, \mathrm{H}-\mathrm{C}\left(1^{\prime}\right)\right), 4.56-4.43(\mathrm{~m}, 2 \mathrm{H}), 4.34-4.23(\mathrm{~m}, 2 \mathrm{H}), 3.86(\mathrm{br} d, 1 \mathrm{H}, J=11.6)$, $3.10(d d, 1 \mathrm{H}, J=11.1,4.8, \mathrm{H}-\mathrm{C}(3)), 2.77$ ( $b r d, 1 \mathrm{H}, J=10.9$, H-C(18)), 0.93, 0.85, 0.82, 0.73, 0.73, 0.62, $0.41\left(s, 7 \times 3 \mathrm{H}, \mathrm{CH}_{3}\right)$; ESI-MS: $1501.9\left[(\mathrm{M}+\mathrm{Na})^{+}\right]$.

Compound 13b (396 mg, $0.268 \mathrm{mmol}$ ) was dissolved in dry $\mathrm{CH}_{2} \mathrm{Cl}_{2}-\mathrm{MeOH}(1: 2,30 \mathrm{~mL})$, to which a freshly prepared solution of $\mathrm{NaOMe}$ in $\mathrm{MeOH}(1.0 \mathrm{M}, 1.20 \mathrm{ml})$ was added. The mixture was stirred at $\mathrm{rt}$ for $3 \mathrm{~h}$ and then neutralized with Dowex $\mathrm{H}^{+}$resin to $\mathrm{pH}$ 7. The resin was then removed by filtration. Recrystallization with $\mathrm{MeOH}_{-} \mathrm{Et}_{2} \mathrm{O}$ furnished $\beta$-D-glucopyranosyl oleanolate 3-O- $\alpha$-Larabinopyranoside (14b) $(133 \mathrm{mg}, 66 \%)$ as a white powder. $R_{\mathrm{f}}=0.46\left(4: 1, \mathrm{CHCl}_{3}-\mathrm{MeOH}\right) ;{ }^{1} \mathrm{H}-\mathrm{NMR}$ (pyridine- $\left.d_{5}\right) \delta 6.33\left(d, 1 \mathrm{H}, J=7.9, \mathrm{H}-\mathrm{C}\left(1^{\prime \prime}\right)\right), 5.42(b r s, 1 \mathrm{H}, \mathrm{H}-\mathrm{C}(12)), 4.75\left(d, 1 \mathrm{H}, J=7.1, \mathrm{H}-\mathrm{C}\left(1^{\prime}\right)\right)$, 4.45-4.37 (m, 4H), 4.33-4.28 (m, 3H), 4.17-4.13 (m, 2H), $4.03(m, 1 \mathrm{H}), 3.82(b r d, 1 \mathrm{H}, J=10.7), 3.34$ $(m, 1 \mathrm{H}, \mathrm{H}-\mathrm{C}(3)), 3.19(d d, 1 \mathrm{H}, J=11.9,2.0, \mathrm{H}-\mathrm{C}(18)), 1.26,1.24,1.09,0.94,0.90,0.88,0.86(s, 7 \times 3 \mathrm{H}$, $\mathrm{CH}_{3}$ ); ${ }^{13} \mathrm{C}-\mathrm{NMR}$ (pyridine- $d_{5}$ ) $\delta 176.4,144.1,122.9,107.5,95.8,88.7,79.3,78.9,74.6,74.2,72.9$, 71.2, 69.5, 66.7, 62.3, 55.9, 48.1, 47.1, 46.3, 42.2, 41.8, 39.9, 39.6, 38.9, 37.1, 34.1, 33.2, 33.1, 32.6, $30.8,28.3,28.3,26.6,26.1,23.9,23.7,23.5,18.6,17.5,16.9,15.6$; ESI-MS: $785.2\left[(\mathrm{M}+\mathrm{Cl})^{-}\right]$. HRMS: $m / z 750.4485[\mathrm{M}-\mathrm{H}]^{-}\left(\left[\mathrm{C}_{41} \mathrm{H}_{65} \mathrm{O}_{12}\right]=750.4481\right)$.

$\beta$-D-Glucopyranosyl oleanolate 3-O- $\beta$-D-galactopyranoside (14a).

This substance was prepared according to the same procedure described for $14 \mathbf{b} . R_{\mathrm{f}}=0.24$ (4:1, $\mathrm{CHCl}_{3}-\mathrm{MeOH}$ ); ${ }^{1} \mathrm{H}-\mathrm{NMR}$ (pyridine- $\left.d_{5}\right) \delta 6.36(d, 1 \mathrm{H}, J=7.9, \mathrm{H}-\mathrm{C}(1 ")), 5.45$ (br s, 1H, H-C(12)), 4.88 
$\left(d, 1 \mathrm{H}, J=7.7, \mathrm{H}-\mathrm{C}\left(1^{\prime}\right)\right), 4.61(d, 1 \mathrm{H}, J=3.2), 4.51-4.43(m, 5 \mathrm{H}), 4.37-4.25(m, 3 \mathrm{H}), 4.20-4.15(m, 2 \mathrm{H})$, $4.06(m, 1 \mathrm{H}), 3.39(d d, 1 \mathrm{H}, J=11.6,3.6, \mathrm{H}-\mathrm{C}(3)), 3.22(d d, 1 \mathrm{H}, J=13.2,2.4, \mathrm{H}-\mathrm{C}(18)), 1.32,1.29$, $1.12,0.98,0.93,0.90,0.86\left(s, 7 \times 3 \mathrm{H}, \mathrm{CH}_{3}\right) ;{ }^{13} \mathrm{C}-\mathrm{NMR}$ (pyridine- $\left.d_{5}\right) \delta 176.4,144.2,122.9,107.5,95.8$, $88.8,79.3,78.9,76.8,75.5,74.2,73.2,71.2,70.3,62.5,62.3,55.9,48.1,47.0,46.3,42.2,41.8,40.0$, 39.6, 38.8, 37.0, 34.1, 33.2, 33.2, 32.6, 30.8, 28.3, 28.3, 26.7, 26.2, 23.8, 23.7, 23.5, 18.6, 17.5, 17.0, 15.6; ESI-MS: $781.0\left[(\mathrm{M}+\mathrm{H})^{+}\right], 1561.1\left[(2 \mathrm{M}+\mathrm{H})^{+}\right]$. HRMS: $m / z 779.4580[\mathrm{M}-\mathrm{H}]^{-}\left(\left[\mathrm{C}_{42} \mathrm{H}_{67} \mathrm{O}_{13}\right]=\right.$ 779.4587).

Synthesis of $O A$ 3,28-diglycosides 16a 16b, approach 2: $\beta$-D-xylopyranosyl oleanolate 3-O- $\beta$ - $D$ galactopyranoside (16a).

A mixture of intermediate $\mathbf{1 1 b}(600 \mathrm{mg}, 0.666 \mathrm{mmol})$, trichloroacetimidate $\mathbf{3 b}(570 \mathrm{mg}, 0.765$ mmol) and powdered $4 \AA$ molecular sieves $(600 \mathrm{mg})$ in dry $\mathrm{CH}_{2} \mathrm{Cl}_{2}(9 \mathrm{~mL})$ were stirred for $30 \mathrm{~min}$ at rt. A solution of TMSOTf in dry $\mathrm{CH}_{2} \mathrm{Cl}_{2}(5 \%, 128 \mu \mathrm{L})$ was added dropwise and the mixture was stirred for $30 \mathrm{~min}$ before $\mathrm{Et}_{3} \mathrm{~N}(0.40 \mathrm{ml})$ was added to quench the reaction. The mixture was then filtered and the filtrate was concentrated and purified by a silica gel column chromatography $(3: 1$, petroleum ether-EtOAc) to afford 2,3,4-tri- $O$-benzoyl- $\beta$-D-xylopyranosyl oleanolate 3-O-(2,3,4,6tetra- $O$-benzoyl- $\beta$-D-galactocopyranoside) (15a) $(920 \mathrm{mg}, 93.4 \%)$ as a white powder. $R_{\mathrm{f}}=0.23(3: 1$, petroleum ether-EtOAc); ${ }^{1} \mathrm{H}-\mathrm{NMR}\left(\mathrm{CDCl}_{3}\right) \delta$ 8.12-7.22 ( $\left.m, 35 \mathrm{H}, \mathrm{H}-\mathrm{C}(\mathrm{Ar})\right), 5.95\left(\mathrm{~s}, 1 \mathrm{H}, \mathrm{H}-\mathrm{C}\left(4^{\prime}\right)\right), 5.90$ $\left(t, 1 \mathrm{H}, J=8.8, \mathrm{H}-\mathrm{C}\left(2^{\prime \prime}\right)\right), 5.87\left(d, 1 \mathrm{H}, J=7.4, \mathrm{H}-\mathrm{C}\left(1^{\prime \prime}\right)\right), 5.83\left(t, 1 \mathrm{H}, J=9.2, \mathrm{H}-\mathrm{C}\left(2^{\prime}\right)\right), 5.64-5.59(m, 2 \mathrm{H}$, H-C(3'), H-C(3")), 5.40 ( $\left.m, 1 \mathrm{H}, \mathrm{H}-\mathrm{C}\left(4^{\prime \prime}\right)\right), 5.30$ (br s, 1H, H-C(12)), 4.81 (d, 1H, J=7.9, H-C(1')), 4.65 ( $\left.d d, 1 \mathrm{H}, J=11.1,7.7, \mathrm{H}-\mathrm{C}\left(6^{\prime}\right)-1\right)$, 4.44-4.41 ( $\left.m, 2 \mathrm{H}, \mathrm{H}-\mathrm{C}\left(6^{\prime}\right)-2, \mathrm{H}-\mathrm{C}\left(5^{\prime \prime}\right)-1\right), 4.30$ ( $\left.m, 1 \mathrm{H}, \mathrm{H}-\mathrm{C}\left(5^{\prime}\right)\right), 3.72$ $(d d, 1 \mathrm{H}, J=14.7,6.8, \mathrm{H}-\mathrm{C}(5 ")-2), 3.09$ (dd, 1H, $J=11.7,4.3, \mathrm{H}-\mathrm{C}(3)), 2.81(b r d, 1 \mathrm{H}, J=10.8, \mathrm{H}-$ $\mathrm{C}(18)), 0.94,0.88,0.87,0.79,0.67,0.66,0.44\left(s, 7 \times 3 \mathrm{H}, \mathrm{CH}_{3}\right)$; ESI-MS: $1478.0\left[(\mathrm{M}-\mathrm{H})^{-}\right], 1501.9$ $\left[(\mathrm{M}+\mathrm{Na})^{+}\right]$.

Compound 15a (340 mg, $0.230 \mathrm{mmol}$ ) was dissolved in dry $\mathrm{CH}_{2} \mathrm{Cl}_{2}-\mathrm{MeOH}(1: 2,36 \mathrm{~mL})$, to which a freshly prepared solution of $\mathrm{NaOMe}$ in $\mathrm{MeOH}(1.0 \mathrm{M}, 1.60 \mathrm{~mL})$ was added. The solution was stirred at $\mathrm{rt}$ for $12 \mathrm{~h}$ and then neutralized with Dowex $\mathrm{H}^{+}$resin to $\mathrm{pH}$ 7. The resin was then removed by filtration. Recrystallization with $\mathrm{MeOH}-\mathrm{Et}_{2} \mathrm{O}$ furnished $\beta$-D-xylopyranosyl oleanolate 3-O- $\beta$-Dgalactopyranoside (16a) $(132 \mathrm{mg}, 76.5 \%)$ as a white powder. $R_{\mathrm{f}}=0.41\left(4: 1, \mathrm{CHCl}_{3}-\mathrm{MeOH}\right) ;{ }^{1} \mathrm{H}-$ NMR (pyridine- $\left.d_{5}\right) \delta 6.22\left(d, 1 \mathrm{H}, J=6.4, \mathrm{H}-\mathrm{C}\left(1^{\prime \prime}\right)\right), 5.43(b r s, 1 \mathrm{H}, \mathrm{H}-\mathrm{C}(12)), 4.86(d, 1 \mathrm{H}, J=7.6, \mathrm{H}-$ $\left.\mathrm{C}\left(1^{\prime}\right)\right), 4.58\left(s, 1 \mathrm{H}, \mathrm{H}-\mathrm{C}\left(4^{\prime}\right)\right), 4.48-4.42$ ( $\left.m, 3 \mathrm{H}, \mathrm{H}-\mathrm{C}\left(2^{\prime}\right), \mathrm{H}-\mathrm{C}\left(6^{\prime}\right)-1, \mathrm{H}-\mathrm{C}\left(3^{\prime \prime}\right)\right), 4.36(d d, 1 \mathrm{H}, J=11.6$, 4.3, H-C(5")-1), 4.22 ( $\left.m, 1 \mathrm{H}, \mathrm{H}-\mathrm{C}\left(4^{\prime \prime}\right)\right)$, 4.21-4.15 (m, 3H, H-C(3'), H-C(6')-2, H-C(2")), 4.12 ( $m, 1 \mathrm{H}$, $\left.\mathrm{H}-\mathrm{C}\left(5^{\prime}\right)\right), 3.80\left(m, 1 \mathrm{H}, \mathrm{H}-\mathrm{C}\left(5^{\prime \prime}\right)-2\right), 3.36(d d, 1 \mathrm{H}, J=18.7,6.0, \mathrm{H}-\mathrm{C}(3)), 3.23(b r d, 1 \mathrm{H}, J=10.4, \mathrm{H}-$ $\mathrm{C}(18)), 1.29,1.25,1.06,0.96,0.92,0.90,0.83\left(s, 7 \times 3 \mathrm{H}, \mathrm{CH}_{3}\right) ;{ }^{13} \mathrm{C}-\mathrm{NMR}$ (pyridine- $\left.d_{5}\right) \delta 176.6,144.1$, 122.9, 107.6, 96.3, 88.7, 78.4, 76.9, 75.5, 73.7, 73.2, 70.9, 70.3, 67.8, 62.5, 55.8, 48.0, 47.1, 46.2, 42.1, 41.7, 39.9, 39.5, 38.7, 36.9, 34.0, 33.1, 33.1, 32.7, 30.8, 28.2, 28.2, 26.7, 26.1, 23.8, 23.6, 23.3, 18.5, 17.5, 17.0, 15.5; ESI-MS: $773.4\left[(\mathrm{M}+\mathrm{Na})^{+}\right]$. HRMS: $m / z 750.4483[\mathrm{M}-\mathrm{H}]^{-}\left(\left[\mathrm{C}_{41} \mathrm{H}_{65} \mathrm{O}_{12}\right]=750.4481\right)$.

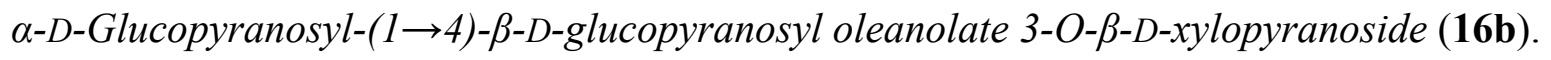

This compound was prepared according to the same procedure described for 16a. $R_{\mathrm{f}}=0.19$ (4:1, $\left.\mathrm{CHCl}_{3}-\mathrm{MeOH}\right) ;{ }^{1} \mathrm{H}-\mathrm{NMR}$ (pyridine- $\left.d_{5}\right) \delta 6.22\left(d, 1 \mathrm{H}, J=8.2, \mathrm{H}-\mathrm{C}\left(1^{\prime \prime}\right)\right), 5.94\left(d, 1 \mathrm{H}, J=3.5, \mathrm{H}-\mathrm{C}\left(1^{\prime \prime \prime}\right)\right)$, $5.42(b r s, 1 \mathrm{H}, \mathrm{H}-\mathrm{C}(12)), 4.82\left(d, 1 \mathrm{H}, J=7.5, \mathrm{H}-\mathrm{C}\left(1^{\prime}\right)\right), 4.58-4.53(m, 3 \mathrm{H}), 4.45(m, 2 \mathrm{H}), 4.37(m, 3 \mathrm{H})$, 
$4.33(t, 1 \mathrm{H}, J=5.8), 4.22(m, 1 \mathrm{H}), 4.17-4.14(m, 4 \mathrm{H}), 4.01(t, 1 \mathrm{H}, J=8.2), 3.87(b r d, 1 \mathrm{H}, J=8.2), 3.77$ $(t, 1 \mathrm{H}, J=11.5), 3.34(d d, 1 \mathrm{H}, J=11.7,4.2, \mathrm{H}-\mathrm{C}(3)), 3.17$ (dd, 1H, $J=10.9,3.9, \mathrm{H}-\mathrm{C}(18)), 1.29,1.25$, 1.07, 0.98, 0.90, 0.88, $0.86\left(s, 7 \times 3 \mathrm{H}, \mathrm{CH}_{3}\right) ;{ }^{13} \mathrm{C}-\mathrm{NMR}$ (pyridine- $\left.d_{5}\right) \delta 176.4,144.1,123.0,107.8$, 103.1, 95.5, 88.7, 80.6, 78.7, 78.3, 77.6, 75.6, 75.4, 75.4, 74.5, 73.6, 71.9, 71.3, 67.2, 62.8, 61.4, 55.9, $48.1,47.0,46.2,42.2,41.8,40.0,39.6,38.8,37.1,34.0,33.2,33.2,32.6,30.8,28.2,28.2,26.8,26.1$, 23.9, 23.7, 23.4, 18.5, 17.5, 17.0, 15.6; ESI-MS: 935.5 [(M+Na) $\left.{ }^{+}\right]$. HRMS: $m / z$ 911.5011 [M-H] $\left(\left[\mathrm{C}_{47} \mathrm{H}_{75} \mathrm{O}_{17}\right]=911.5011\right)$.

\section{Acknowledgements}

The authors thank The National Natural Science Foundation of China (No. 20472054, 30772641) for financial support.

\section{References}

1. Dzubak, P.; Hajduch, M.; Vydra, D.; Hustova, A.; Kvasnica, M.; Biedermann, D.; Markova, L.; Urban, M.; Sarek, J. Pharmacological activities of natural triterpenoids and their therapeutic implications. Nat. Prod. Rep. 2006, 23, 394-411.

2. Liu, J. Pharmacology of oleanolic acid and ursolic acid. J. Ethnopharmacol. 1995, 49, 57-68.

3. Hsu, H. Y.; Yang, J. J.; Lin, C. C. Effects of oleanolic acid and ursolic acid on inhibiting tumor growth and enhancing the recovery of hematopoietic system postirradiation in mice. Cancer Lett. 1997, 111, 7-13.

4. Barthomeuf, C.; Debiton, E.; Mshvidadze, V.; Kemertelidze, E.; Balansard, G. In vitro activity of hederacolchisid A1 compared with other saponins from Hedera colchica against proliferation of human carcinoma and melanoma cells. Planta Med. 2002, 68, 672-675.

5. Jung, H. J.; Lee, C. O.; Lee, K. T.; Choi, J.; Park, H. J. Structure-activity relationship of oleanane disaccharides isolated from Akebia quinata versus cytotoxicity against cancer cells and NO inhibition. Biol. Pharm. Bull. 2004, 27, 744-747.

6. Li, Y.; Matsuda, H.; Yoshikawa, M. Effects of oleanolic acid glycosides on gastrointestinal transit and ileus in mice. Bioorg. Med. Chem. 1999, 7, 1201-1205.

7. Hardegger, E.; Leemann, H. J.; Robinet, F. G. Steroid and triterpene glycosides. III. Glucosides of esters of oleanic acid and glycosidal binding in beet-sugar saponins. Helv. Chim. Acta. 1952, 35, 824-829.

8. Kochetkov, N. K.; Khorlin, A. Ya.; Snyatkova, V. I. Triterpenoid saponins 13. Halolysis of glycosides of the triterpene series and the synthesis of glucosides of oleanolic acid. Izvestiya Akademii Nauk SSSR, Seriya Khimicheskaya. 1964, 2028-36; English Translation: Russian Chemical Bulletin 1964, 13, 1925-1931.

9. Kochetkov, N. K.; Khorlin, A. Ya.; Bochkov, A. F.; Kretsu, L. G. Synthesis of glycosides of oleanolic acid. Izvestiya Akademii Nauk SSSR, Seriya Khimicheskaya. 1966, 2028-2029; English Translation: Russian Chemical Bulletin 1966, 15, 1963-1965.

10. Juodvirsis, A.; Troshchenko, A. T. Synthesis of triterpene glycosides I. Synthesis of acyl glycosides of oleanolic and ursotic acids. Khimiya Prirodnykh Soedinenii. 1966, 2, 405-410; English Translation: Chemistry of Natural Compounds 1966, 2, 331-334. 
11. Janiszowska, W.; Wilkomirski, B.; Kasprzyk, Z. Synthesis of oleanolic acid 3-O-monoglucoside. Polish Journal of Chemistry. 1980, 54, 2147-2152.

12. Seebacher, W.; Haslinger, E.; Rauchensteiner, K.; Jurenitsch, J.; Presser, A.; Weis, R. Synthesis and Haemolytic Activity of Randianin Isomers. Monatshefte fuer Chemie. 1999, 130, 887-897.

13. Seebacher, W.; Weis, R.; Jurenitsch, J.; Rauchensteiner, K.; Haslinger, E. Synthesis and hemolytic properties of arvensoside B isomers. Monatshefte fuer Chemie. 2000, 131, 985-996.

14. Ohtani, K.; Mizutani, K.; Kasai, R.; Tanaka, O. Selective cleavage of ester type glycosidelinkages and its application to structure determination of natural oligoglycosides. Tetrahedron Lett. 1984, 25, 4537-4540.

15. Deng, S. J.; Yu, B.; Xie, J. M.; Hui, Y. Z. A highly efficient glycosylation of sapogenins. J. Org. Chem. 1999, 64, 7265-7266.

16. Schmidt, R. R.; Michel, J. Facile synthesis of $\alpha$ - and $\beta$-O-glycosyl imidates; Preparation of glycosides and disaccharides. Angew. Chem. Int. Ed. Engl. 1980, 19, 731-732.

17. Zang, J.; Li, Y. X.; Li, C. X.; Song, N.; Yue, C. L. Synthesis of Four Oleanolic Saponins. Periodical of Ocean University of China. 2005, 35, 635-640.

18. Sun, J.; Han, X.; Yu, B. Synthesis of a typical $N$-acetylglucosamine containing saponin, oleanolic acid 3- $O$ - $\alpha$-L-arabinopyranosyl- $(1 \rightarrow 2)$ - $\alpha$-L-arabinopyranosyl-( $(1 \rightarrow 6)$-2-acetamido-2-deoxy- $\beta$-Dglucopyranoside. Carbohydr. Res. 2003, 338, 827-833.

19. Cheng, M. S.; Yan, M. C.; Liu, Y.; Zheng, L. G.; Liu, J. Synthesis of beta-hederin and Hederacolchiside A. 1: triterpenoid saponins bearing a unique cytotoxicity-inducing disaccharide moiety. Carbohydr. Res. 2006, 341, 60-67.

20. Li, C. X.; Zang, J.; Wang, P.; Zhang, X. L.; Guan, H. S.; Li, Y. X. Synthesis of two natural oleanolic acid saponins. Chinese J. Chem. 2006, 24, 509-517.

21. Yu, B.; Xie, J.; Deng, S.; Hui, Y. First Synthesis of a bidesmosidic triterpene saponin by a highly efficient procedure. J. Am. Chem. Soc. 1999, 121, 12196-12197.

22. Peng, W.; Sun, J.; Lin, F.; Han, X.; Yu, B. Facile Synthesis of Ginsenoside Ro. Synlett 2004, 259262.

23. Yan, M. C.; Liu, Y.; Chen, H.; Ke, Y.; Xu, Q. C.; Cheng, M. S. Synthesis and antitumor activity of two natural $\mathrm{N}$-acetylglucosamine-bearing triterpenoid saponins: Lotoidoside D and E. Bioorg. Med. Chem. Lett. 2006, 16, 4200-4204.

24. Winterstein, A.; Stein, G. Double bond between C-12 and C-13 of oleanolic acid is inert to catalytic hydrogenation. Z. Physiol. Chem. 1931, 202, 222-231.

25. Bliard, C.; Massiot, G.; Nazabadioko, S. Glycosylation of acids under phase transfer conditions. Partial synthesis of saponins. Tetrahedron Lett. 1994, 35, 6107-6108.

26. Schmidt, R. R.; Kinzy, W. Anomeric-oxygen activation for glycoside synthesis: The trichloroacetimidate method. Adv. Carbohydr. Chem. Biochem. 1994, 50, 21-125.

Sample Availability: Samples are available from the authors.

(C) 2008 by the authors; licensee Molecular Diversity Preservation International, Basel, Switzerland. This article is an open-access article distributed under the terms and conditions of the Creative Commons Attribution license (http://creativecommons.org/licenses/by/3.0/). 